\title{
Comparison of Factorial Kriging Analysis Method and Upward Continuation Filter to Recognize Subsurface Structures - A Case Study: Gravity Data from a Hydrocarbon Field in the Southeast Sedimentary Basins of the East Vietnam Sea
}

\author{
Mohammad-Reza AZAD ${ }^{1}$, Mohammad KONESHLOO ${ }^{1,2}$, \\ Abolghasem KAMAKAR ROUHANI ${ }^{1}$, and Hamid AGHAJANI ${ }^{1}$ \\ ${ }^{1}$ Shahrood University, Faculty of Mining and Petroleum Engineering \\ and Geophysics, Shahrood, Iran \\ ${ }^{2}$ Department of Chemical and Petroleum Engineering, University of Wyoming, \\ Laramie, Wyoming, USA; e-mail: mkoneshl@uwyo.edu
}

\section{Abstract}

To interpret geophysical anomaly maps, it is necessary to filter out regional and sometimes noise components. Each measured value in a gravity survey consists of different components. Upward continuation (UC) is one of the most widely used filters. The shortcoming of this filter is not to consider the spatial structure of the data, and also the fact that the trial and error approach and expert's judgment are needed to adjust it. This study aims to compare the factorial kriging analysis (FKA) and UC filters for separation of local and regional anomalies in the gravity data of a hydrocarbon field in the southeast sedimentary basins of the East Vietnam Sea. As shown in this paper, FKA method permits to filter out all of the identified structures, while the UC filter does not possess this capability. Therefore, beside general and classic filtering methods, the 
FKA method can be used as a strong method in filtering spatial structures and anomaly component.

Key words: anomaly separation, geophysical filtering, factorial kriging analysis (FKA), upward continuation (UC), hydrocarbon exploration.

\section{INTRODUCTION}

The gravity method is a very useful method in oil, gas, and mineral exploration. Despite the vast use of seismic method in oil exploration, the gravity method is always one of the initial steps in hydrocarbon exploration to recognize favourable areas. This method is usually applied before conducting next exploration steps using advanced methods such as seismic ones and drilling. Considering the fact that the density of oil-gas environments is less than their surroundings, the resulting gravity anomalies can be detected. It has also a realistic significance in discovering sedimentary basins, local structures, and areas with an oil-gas potential (Berezkin 1973, McCulloh 1980, Zeng et al. 2002, Tran 2004, Aghajani et al. 2011).

It is very important in the interpretation of gravity data for hydrocarbon exploration to recognize a sedimentary basin that can be a possible hydrocarbon area (Reynolds 1997). In addition, we know that the Bouguer anomaly maps contain three components: noise, regional, and local anomalies (Telford et al. 1990). Many different methods with varying methodology bases (Nettleton 1954) are used for separation of regional from residual (local) anomalies in order to distinguish areas that may be suitable for the formation of hydrocarbon reservoirs. In fact, these methods separate the local anomaly related to the oil-gas reservoir from regional anomaly.

One of the most widely known methods to extract regional anomaly is called the upward continuation (UC) method that is based on the weighted averages. The weights are calculated using upward continuation level (for details see Telford et al. 1990). UC can be regarded as estimated airborne survey using the ground survey. When the UC method is applied to a ground survey dataset, in fact, the data are converted as if taken from an airborne survey in an upward height or level above the ground survey. There are some problems with the method, such as the fact that the height should be known when using the method Zeng et al. (2007).

Factorial kriging analysis (FKA) is a variogram-based filtering technique (Matheron 1982). FKA is used to filter data with different spatial structures (Sandjivy and Galli 1984, Sandjivy 1984). Galli applied this method as a substitute to spectral analysis of magnetic data (Galli et al. 1984). Seguret (1993) has used FKA to extract diurnal variation from aeromagnetic data. Moreover, some experts used the FKA to reorganize geological structures on 
gravity data, and also to filter seismic noisy data (Jeannée and Mari 2008, Magneron et al. 2009).

This work aims to present theory of the FKA method and its application to separate gravity anomalies in a hydrocarbon field in the southeast sedimentary basins of the East Vietnam Sea. After a brief description of the study area, the variographic study of data is presented, then the FKA and UC results are demonstrated, and finally the results of the two methods are compared considering the geological features of this hydrocarbon field.

\section{THEORY OF THE USED METHODS}

\subsection{FKA}

Conventional methods (e.g., UC method) have some practical limitations; firstly, a preliminary interpolation (e.g., kriging) is needed to estimate the value of variable on a complete rectangular grid, when data are not on a regular grid. Secondly, their interpretation and implementation become tedious by increasing the dimension of space, and finally, by ignoring the spatial variation of the component of frequency, a local anomaly can widespread through all frequencies (Chilès and Delfiner 1999). To solve this problem, Matheron (1982) proposed an orthogonal decomposition of a regionalized variable $Z(x)$ to its components $Z_{s}(x)$. That means, a random function as $Z(x)$ can be assumed as an intrinsic regionalization model with mostly stationary components, in which $Z_{s}(x)$ is an intrinsic component (Wackernagel 1988). In this case,

$$
\gamma(h)=\gamma\left(x_{\alpha}-x_{\beta}\right)=E\left\{\left[Z\left(x_{\alpha}\right)-Z\left(x_{\beta}\right)\right]^{2}\right\}=E\left\{\left[\sum_{i=1}^{s} Z^{i}\left(x_{\alpha}\right)-\sum_{i=1}^{s} Z^{i}\left(x_{\beta}\right)\right]^{2}\right\},
$$

where $x_{\alpha}$ and $x_{\beta}$ represent the location of any point of $\alpha$ and $\beta, Z\left(x_{\alpha}\right)$ and $Z\left(x_{\beta}\right)$ are the measured values at these points. If the structures are assumed to be mutually uncorrelated, then this formula can be written as a summation of the components' variograms.

$$
\gamma(h)=E\left\{\sum_{i=1}^{s}\left(Z^{i}\left(x_{\alpha}\right)-Z^{i}\left(x_{\beta}\right)\right)^{2}\right\}=\sum_{i=1}^{s} E\left(Z^{i}\left(x_{\alpha}\right)-Z^{i}\left(x_{\beta}\right)\right)^{2}=\sum_{i=1}^{s} \gamma^{i}\left(x_{\alpha}-x_{\beta}\right) .
$$

This kind of spatial structure is called nested variogram model, as schematically shown in Fig. 1. Matheron (1982) demonstrated the formula of this new method, named FKA, in the case of monovariable and also developed it for the case of heterotopic multivariable. The spatial structure, characterized by a covariance/variogram model, is a space domain equivalent of density spectrum in the frequency domain (Yao 2004); thus, FKA relies on the de- 
(a)

(b)
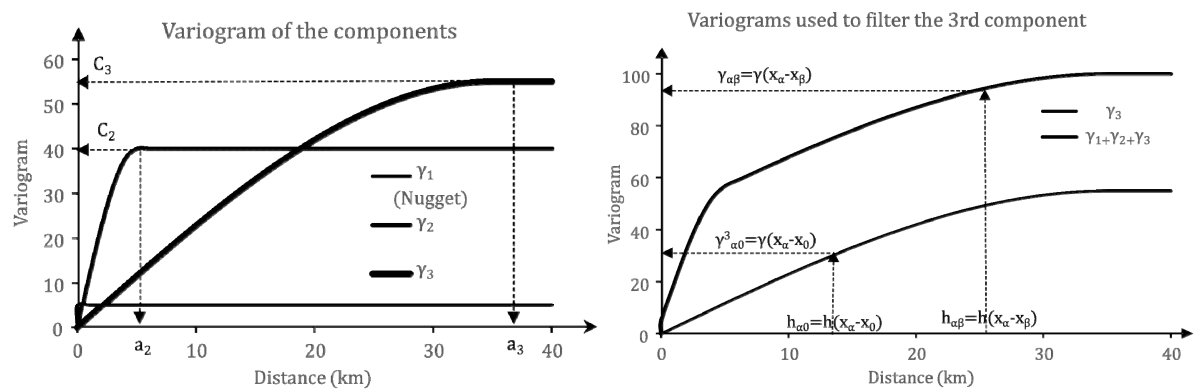

Fig. 1. Each measured gravity value of $Z(x)$ could be assumed as the summation of three different orthogonal components; as an example: noise or very short scale component as $Z^{1}(x)$, local as $Z^{2}(x)$, and regional component as $Z^{3}(x)$, where $Z(x)=Z^{1}(x)+Z^{2}(x)+Z^{3}(x)$; (a) the range and sill of variograms are shown, respectively, by $a_{i}$ and $C_{i}$, the first structure is a pure nugget effect and has no range; (b) overall variogram is used in the right hand matrix of kriging to calculate variogram value between the known points and variogram of 3rd component (to be filtered) is used to complete the right hand matrix of kriging including the variogram between the known points and target.

composition of variograms, which permits to overpass the above-mentioned limitations for conventional methods for interpretation of field potential data.

Goovaerts (1997) has presented FKA in "dual form" and gave an example of the filtering properties of kriging. Wackernagel (1995) demonstrated the FKA formula to filter out the intrinsic and stationary components of a regionalized variable. An example for univariate case is the measured value in a gravity survey; the measured value could be factorized to its components which have different wavelengths. Value of intrinsic component at each point of $x_{0}$ denoted as $Z\left(x_{0}\right)$ can be estimated using the values of the measured variable in a given neighbourhood $Z\left(x_{\alpha}\right)$ :

$$
Z_{s}^{*}\left(x_{0}\right)=\sum_{\alpha=1}^{n} w_{\alpha}^{s} Z\left(x_{\alpha}\right)
$$

The reader is referred to Wackernagel $(1988,1995)$, Goovaerts (1997, page 165-166), and Chilès and Delfiner (1999, pages 342-345) for details of the mathematical framework of this method.

1. Non bias condition: The expectation of the estimation error should be nil;

2. Minimizing the variance of error;

3. Non-correlation condition between components. 


$$
\begin{aligned}
\sigma_{E}^{2}= & \sum_{u=0}^{s-1} C^{u}\left(x_{\alpha}-x_{\beta}\right)-\gamma^{s}\left(x_{\alpha}-x_{\beta}\right)-\sum_{\alpha=1}^{n} \sum_{\beta=1}^{n} w_{\alpha}^{s} w_{\beta}^{s} \gamma^{s}\left(x_{\alpha}-x_{\beta}\right)+2 \sum_{\alpha=1}^{n} w_{\alpha}^{s} \gamma^{s}\left(x_{\alpha}-x_{0}\right), \\
& \sum_{\beta=1}^{n} w_{\beta}^{s} \gamma\left(x_{\alpha}-x_{\beta}\right)+\mu_{s}=\gamma^{s}\left(x_{\alpha}-x_{0}\right) \text { for } \alpha=1,2, \ldots, n, \\
& \sum_{\beta=1}^{n} w_{\beta}^{s}=1 .
\end{aligned}
$$

where $w_{\beta}^{s}$ represents the weight of kriging for each point of $x_{\beta}, \gamma\left(x_{\alpha}, x_{\beta}\right)$ is the value of variogram between each two pairs of points of $x_{\alpha}$ and $x_{\beta}$; in the same way, $\gamma^{s}\left(x_{\alpha}, x_{0}\right)$ is equal to the value of variogram for the distance between $x_{\alpha}$ and $x_{0}$ (see Fig. 1b), and finally $\mu_{s}$ is the LaGrange multiplier.

The filtering system, expressed by Eq. 5, is similar to the system of ordinary kriging, but the variogram value of right-hand side matrix of this equation, $\gamma^{s}\left(x_{\alpha}, x_{0}\right)$, is calculated only using the spatial structure that must be filtered.

\subsection{UC}

There are many different methods concerned with separation of the regional and local or residual anomalies in geophysics, especially in gravity and magnetic geophysical techniques. The UC method is used frequently to identify regional anomalies and gravity/magnetic variations of deeper horizons (Claerbout 1986). This method is applied to filter out short-wavelength (or high-frequency) anomalies, and also, to reduce their amplitudes and decrease the noise related by near surface features (Reynolds 1997). This filter or operator is a transformation of the potential field anomaly calculated at a height greater than the observed field $A(x, y, z)$, that is, a measured or observed potential field at a location $(x, y, z)$ in space. If there is no source in the upper half space, then (Gibert and Galdeano 1985):

$$
\Delta[A(x, y, z)]=0 \quad \text { where } \quad z \geq 0 .
$$

Potential field in different points is expressed as follows (Jacobsen 1987):

$$
A(x, y, z)=\frac{z}{2 \pi} \int_{-\infty-\infty}^{\infty} \int_{[}^{\infty} \frac{A(\xi, \eta, 0)}{\left[(x-\xi)^{2}+(y-\eta)^{2}+z^{2}\right]^{3 / 2}} d \xi d \eta,
$$

where $\xi$ and $\eta$ are the coordinates. Equation 7 can be written as a convolution (Gibert and Galdeano 1985) given by Eq. 8: 


$$
A(x, y, z)=A(x, y, 0) * P_{z}(x, y),
$$

where * denotes convolution; hence, the Fourier transformation of Eq. 8 is equal to the Fourier transform of the first term multiplied by the Fourier transform of the second terms (Everett 2013), which is

$$
P_{z}(x, y)=\frac{z}{2 \pi\left(x^{2}+y^{2}+z^{2}\right)^{3 / 2}},
$$

$A(x, y, 0)$ is the upward continued potential field, i.e., the potential field taken to the height 0 , that is, higher than the height $z$ related to the location of the observed or measured field $A(x, y, z)$; it should be noted that the direction of $z$ axis downward is positive.

The UC operator is a filter that eliminates high frequency components and, in contrast, the low-frequency components will be strengthened. In particular, the UC filter tries to filter out the shorter wavelength anomalies that are regarded as near surface structures, and the subsequent process is highlighting the effect of deep subsurface structures. Although one of the most widely used filtering techniques in the processing and interpretation of potential field data is the UC method, there are some disadvantages too, the most important being the fact that it does not consider the spatial structure of the data, and also the lack of knowledge about the optimum UC height for a particular case. In this paper, a geostatistical filtering method, called FKA, is used instead of the UC method. Then each of the structures (including anomalies and noise) can be separated using the FKA theory explained in detail in Section 2.1. In the following, we will first briefly review of the study area and then will apply the methods separately, and finally, compare the results.

\section{OVERVIEW OF THE STUDY AREA}

\subsection{Geology of the study area}

The study area is located in the southeastern part of a Vietnamese basin situated in the east of Vietnam Sea (Fig. 2a). The studied field covers an area of $215 \times 215 \mathrm{~km}$. There are numerous oil-gas boreholes drilled in the basin. The prior studies have shown some oil-gas reservoirs developed in the EoceneOligocene and the Early Miocene formations with the marine-river source, but many of them have been concentrated in the broken rock of the PreCenozoic strata. The multiple oil-gas reservoirs are developed at different depths and connected together horizontally. In the area, the thickness of sedimentary layer varies very strongly, from $1 \mathrm{~km}$ along the coastline to $13 \mathrm{~km}$ in the center of the sedimentary basin. Many studies have pointed out that the rocks fill up the grabens in the Eocene-Oligocene strata, which are in 
(a)

(b)
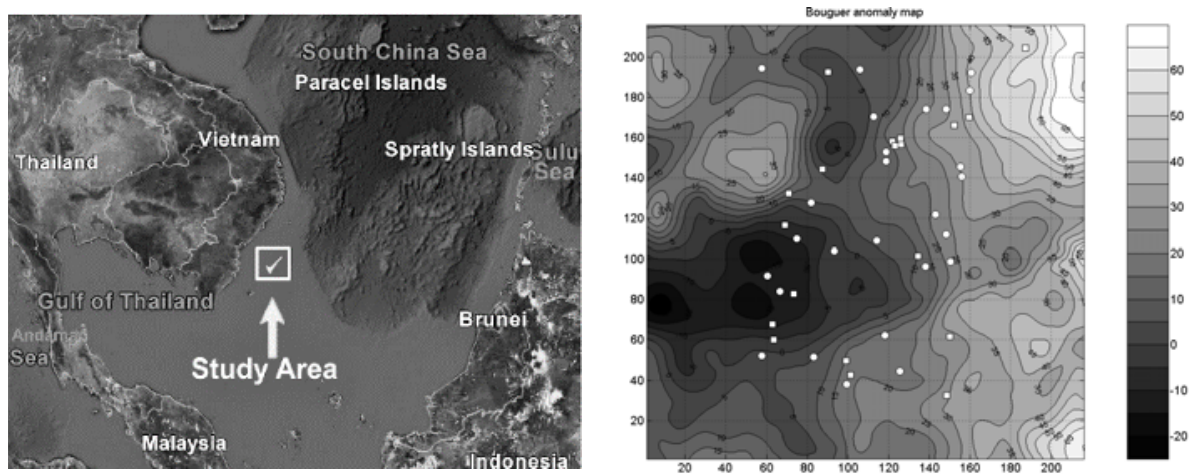

Fig. 2: (a) Location of the $215 \times 215 \mathrm{~km}$ study area (Tran 2004), and (b) Bouguer anomaly map and drilled wells position map of the study area ( $\mathrm{O}-$ wet bore, $\square-$ dry bore). The origin of the map coordinate system is located in the lower-left corner of the map, with positive values extending along the axes up and to the right of the origin point. The values of $X$ and $Y$ coordinates are in $\mathrm{km}$. The contour interval is 5 mGals. Negative Bouguer anomalies are indicated by darker colors.

contact with the horst of carbonate basement rock, and create the potential oil-gas reservoirs (Que and Tran 1996, Vu 2003, Tan and Bo 2002, Tran 2004).

\subsection{Gravity data}

The gravity anomalies have been measured on a very fine grid with a high quality. The gravity map has been drawn with a contour interval of $2 \mathrm{mGals}$, and all the necessary computations in this regard have been performed on a grid of $1 \times 1 \mathrm{~km}$ (Fig. 2b). The used data are drawn by resampling from this exhaustive data base on a grid of $30 \times 30 \mathrm{~km}$ to investigate the capability of the FKA and UC methods on the larger grid. The reason for the increase of the grid size from $1 \times 1 \mathrm{~km}$ to $30 \times 30 \mathrm{~km}$ was the long time of the execution of the FKA computer program when the huge gravity data taken from $1 \times 1 \mathrm{~km}$ grid size was used in the program. However, when the grid size increased, a considerable reduction in the run time was observed due to the reduction of the number of gravity data in the grid. Although different grid sizes were tested, the $30 \times 30 \mathrm{~km}$ grid size containing much less data than $1 \times 1 \mathrm{~km}$ grid size was the largest grid size for which its FKA results were comparable with the UC results. The values of the gravity anomalies vary from -30 to +90 mGals. The negative anomalies indicate sedimentary materials of the basins, and have amplitudes of about tens of mGals lying in the central area but not in the sedimentary basin. It is attributed to the accretion 
of the basement structure. The main trends of the gravity anomalies are northeast-southwest. Some bands of strong gravity gradient are also observed here in northeast-southwest and northwest-southeast directions, some being hundreds kilometers long.

\section{GEOSTATISTICAL FILTERING}

The first step in a geostatistical filtering (FKA) procedure is the spatial structure analysis. This step permits to recognize the components and model them by a nested variogram model in which each component is represented by a simple structure.

\subsection{Structural analysis of the gravity data and variogram modeling}

Variographic study is performed in different directions. Main directions of anisotropy are along N30E and N120E (Figs. 3a, b). Both types of anisotropy, including zonal anisotropy and geometric anisotropy, are observed. If directional variogram shows different effect ranges in several directions, this type of anisotropy is called the geometric anisotropy. Zonal anisotropy can occur when variograms in different directions suggest a different value for the sill (Wackernagel 1988). Once experimental variograms have been calculated along main directions of anisotropy, one should fit a credible model to them. The procedure of analyzing the anisotropy and choosing the anisotropy and fitting the variogram to the model is explained in many geostatistical books (e.g., Armstrong 1998, Isaaks and Srivastava 1989). The best fitted model is a nested model that includes three structures or components indicated in the right hand side of the following equation:

$$
\gamma(h)=10 . \text { Nugget }+170 . \operatorname{Cub}\left(\frac{N 30 E}{145}, \frac{N 120 E}{190}\right)+335 \cdot \operatorname{Cub}\left(\frac{N 30 E}{200}, \frac{N 120 E}{370}\right) .
$$

Each coefficient shows the portion of the component from the total variance of variable, and the number in the parentheses shows the main axes of anisotropy and the value of ranges (see Fig. 3). A cubic variogram model is defined by the following equation (Chilès and Delfiner 1999):

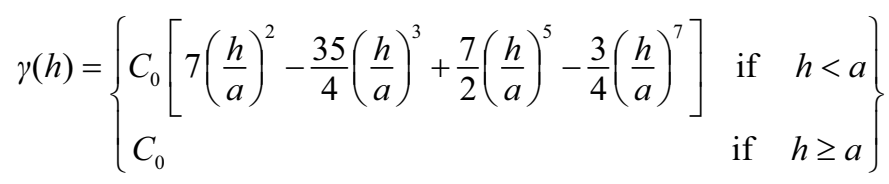

Ellipses of anisotropy are shown in Fig. 3c. The bigger ellipse concerns the larger cubic structure (the third structure indicating a regional anomaly) and the smaller one shows the second structure. 
(a)

(b)
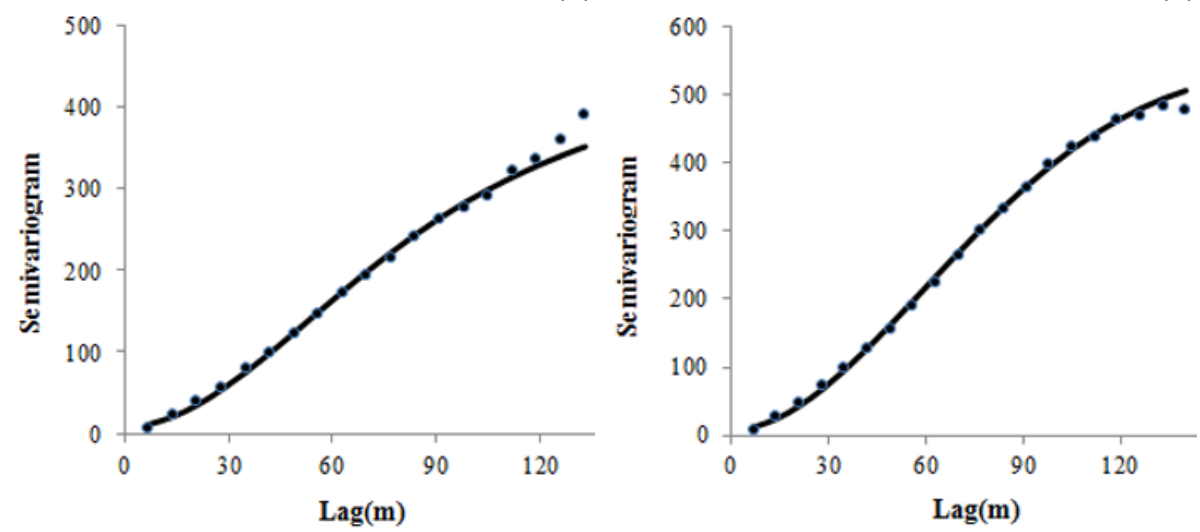

(c)

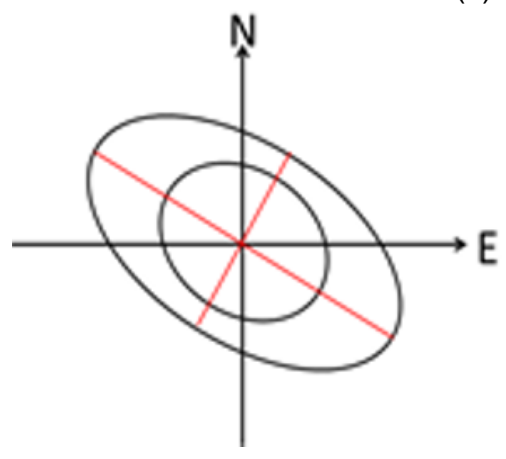

Fig. 3. Experimental and fitted variograms modeled to the gravity data: (a) along N30E, and (b) along N120E. Panel (c) shows the anisotropy ellipse; the radii of ellipses are proportional to the range of their variograms.

The portion of nugget is very small in comparison with two other structures; there are two possible interpretations for a nugget effect:

(i) The distance between gravity stations $(30 \mathrm{~km})$ is too large to provide detailed information about small range models, which may be associated with very high-frequency spatial variations of the gravity data due to very local geologic phenomena;

(ii) Data may be affected by a measurement error, which directly causes a discontinuity in the variogram behavior at the origin. Experimental variogram of data reveals a hierarchical structure, modeled by the mentioned nested structures (Eq. 10), each structure being characterized by its own range and direction. The short range component $(145 \mathrm{~km}$ in $\mathrm{N} 30 \mathrm{E}$ and $190 \mathrm{~km}$ in N120E) corresponds to the local changes in the Bouguer anomaly of the basin, and long-range component may be interpreted as more regional geological or sedimentation changes of the basin. Long-range components seems to be more anisotropic. 


\subsection{Separation of structures in the gravity data by FKA method}

The purpose of this step is to filter out identified spatial components at all nodes of the grid. The 3rd structure has been filtered using Eq. 5. Figure 4a shows the filtered large-scale component results obtained as a consequence of applying FKA method on the gravity data. Then, the values of this component are subtracted from the measured gravity values to obtain the residual values to filter out the second structure. As a result, the short-scale structure component of the gravity data has been shown in Fig. $4 \mathrm{~b}$. The final residual structure has been assumed as the equivalent variable of the nugget effect component (Fig. 4c). The coordinates are in kilometers.

Normally, the regional anomaly is in relation with deep structures effect and the residual anomaly points the near surface structures effect (Reynolds 1997). As can be seen in Fig. 4a, the regional component keeps a decreasing trend from east to west of the study area. An anomaly with low values is notable in the western part of the map of the regional component shown in

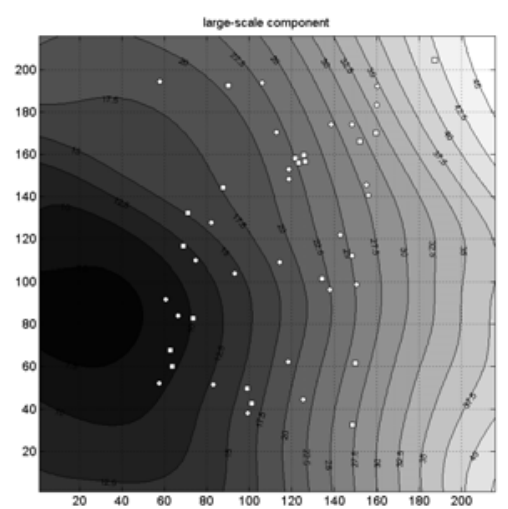

(a)
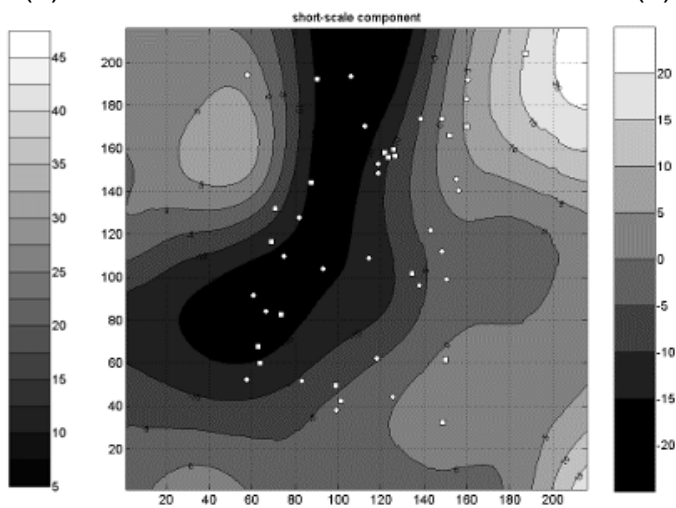

(c)
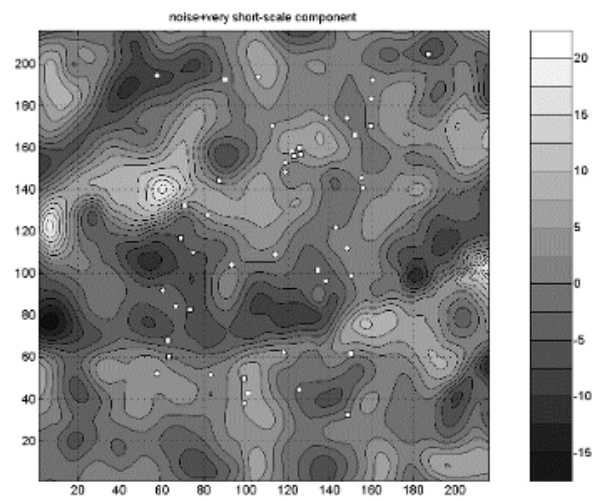

Fig. 4. Maps of different components in the gravity data: (a) large-scale structure, (b) short-scale structure, and (c) nugget and very short-scale effect structure. The values of $X$ and $Y$ coordinates are in kilometers. Contours show the value of Bouguer anomaly. Please note that the color scales are not the same for the components. 
Fig. 4a. That may be interpreted as the effect of low-density sedimentary layers. This structure is distinct from the measured gravity data map. The short-scale component map (Fig. 4b) shows completely different features. High values in this map indicate high-density geological structures such as dense rocks or features present in the area. Moreover, a very notable negative anomaly exists that has been extended from north to the center of the map with a north-southwest trend. This anomaly can be due to the existence of low density sedimentary structures in this part of the area. This part can be regarded as a suitable zone for hydrocarbon reservoirs occurrence, as shown in the previous research by Tran (2004), and the drilled bores often reach to the oil reservoirs in the study area.

\section{FILTERING THE GRAVITY DATA BY UC METHOD}

Tran (2004) and Aghajani (2009) have applied normalized total gradient (NTG) method on the gravity data to detect the gravity anomalies caused by oil-gas reservoirs in the marine sedimentary basins of the study area. Tran (2004) has also pointed out that applying other processing and interpretation techniques on the gravity data is needful to check and enrich the results of NTG method. The UC filter has been applied using different heights of 15 , $20,25,30,35$, and $40 \mathrm{~km}$, and the results of upwarding for $15,20,25$, and $30 \mathrm{~km}$ have been presented as the maps shown in Fig. 5 .

In the interpretation of gravity data for hydrocarbon exploration, it is significant to be able to distinguish between the sedimentary basins which are good possible hydrocarbon targets, and other low-density geological structures that have no prospect for hydrocarbon. These geological structures as well as sedimentary basins produce negative gravity anomalies. In this paper, to determine the depositional areas for possible hydrocarbon occurrence, UC filter with heights of 15, 20,25, and $30 \mathrm{~km}$ have been employed. As can be seen from Fig. 5, the gravity anomalies become smoother, and the anomalies embedded at intermediate depths may even disappear as the height of the UC filter increases.

Hence, we can see the main subsurface structures or anomalies in the maps obtained as a result of applying UC filter with heights of 15, 20, and $25 \mathrm{~km}$; however, the effects of some of these subsurface structures are largely disappeared in the UC map with a height of $30 \mathrm{~km}$. As can also be seen from all of the maps shown in Fig. 5, a lower Bouguer anomaly can be seen in the southwest and west of the study area, and an increase in the Bouguer anomaly is observed toward the east of the area. This low-value Bouguer anomaly can be interpreted as being due to low density geological structures with special geometrical shapes, such as a geo-anticline or salt domes. In contrast, high value Bouguer anomaly can be explained by an increase in the thickness or density sediments, which are located in central and eastern parts 
(a)
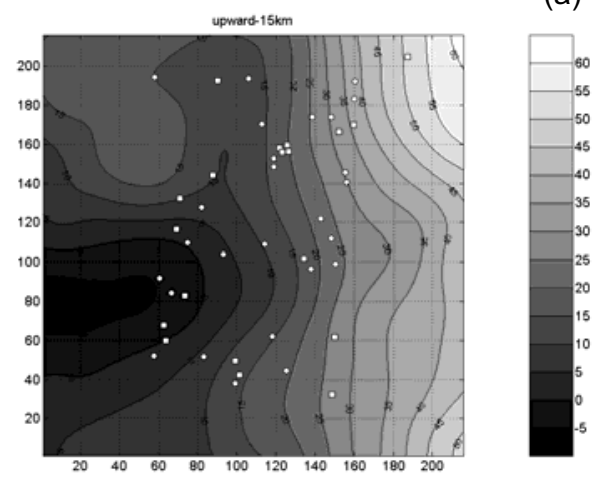

(b)

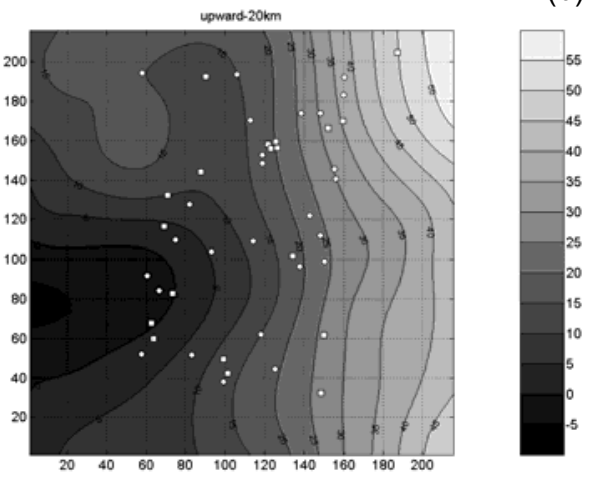

(c)
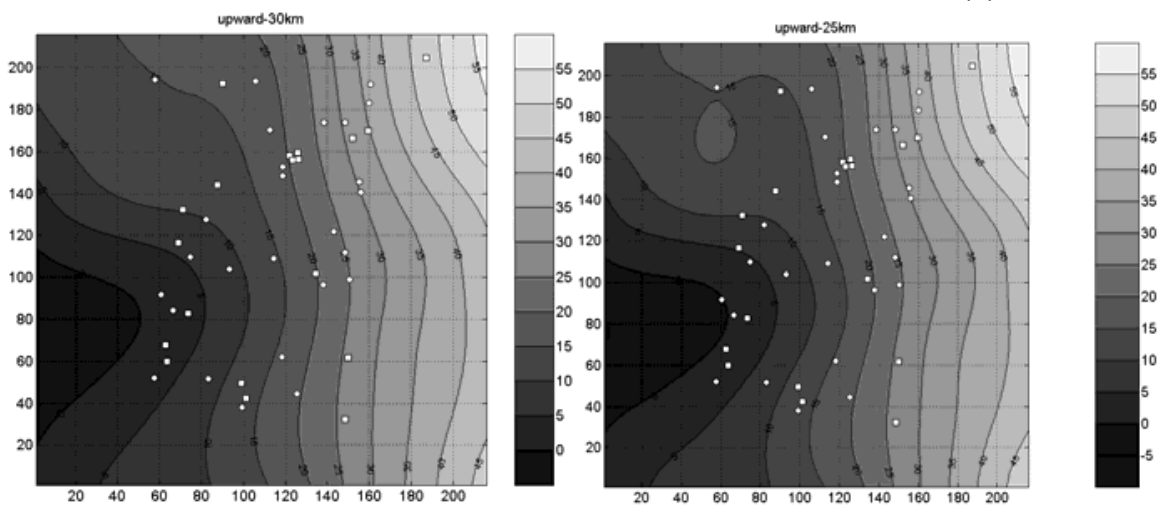

(d)

Fig. 5. The results of applying UC filter for different heights: (a) $15 \mathrm{~km}$, (b) $20 \mathrm{~km}$, (c) $25 \mathrm{~km}$, and (d) $30 \mathrm{~km}$, on the gravity data. The coordinates are in kilometers. Due to different ranges of values, the color scales are not the same.

of the study area. Selection of the height of $25 \mathrm{~km}$ among the other heights seems to be the best or optimum UC height to depict the regional anomaly or anomalies from the gravity data in the area. This is because the effects of the subsurface structures in the UC map obtained with a height greater than $25 \mathrm{~km}$ are the same as those in the UC map with the height of $25 \mathrm{~km}$. Besides, Zeng et al. (2007) introduced a criterion or quantitative method for choosing the optimum UC height. Using this criterion or quantitative method, in which the curve of UC gravity data versus the UC height is drawn to obtain the optimum UC height, the height of $25 \mathrm{~km}$ was approximately obtained. 
If we subtract the obtained regional anomaly from the Bouguer anomaly,
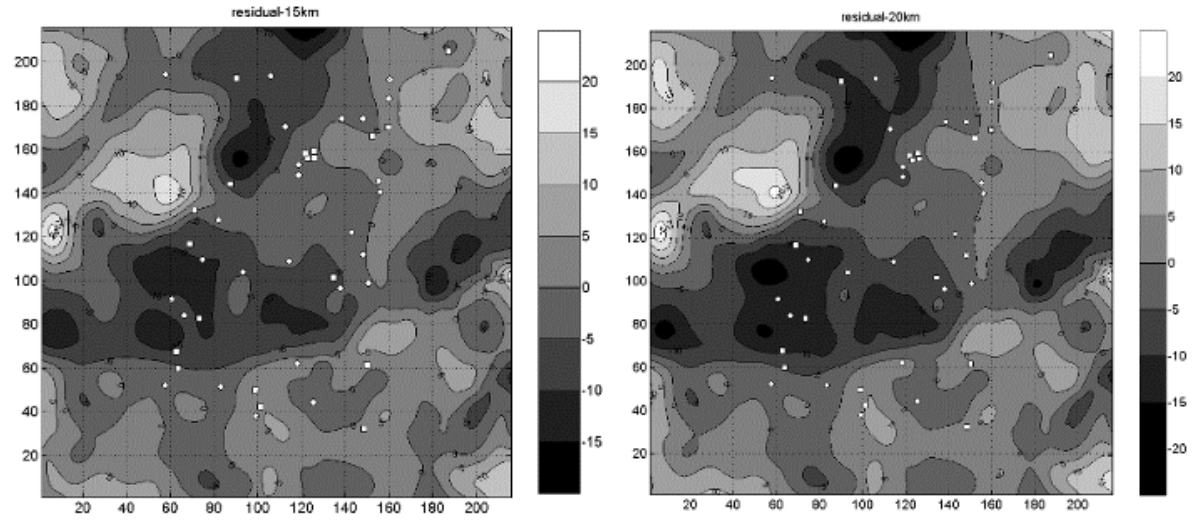

the residual map is obtained, in

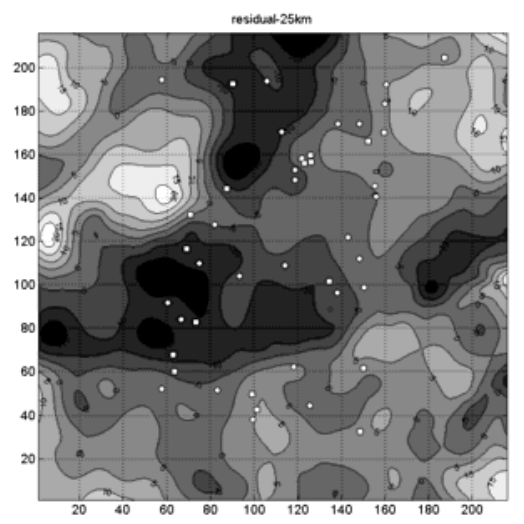

been

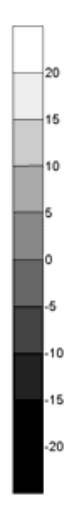

which the local anomalies have

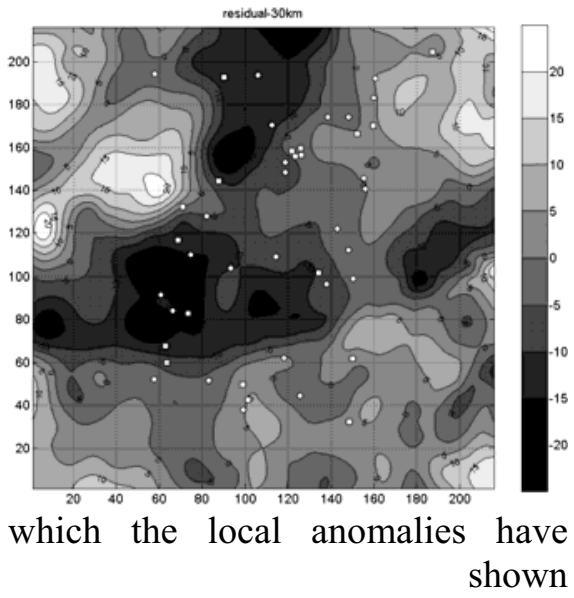

(a)

(c)

Fig. 6. Residual anomalies obtained as a result of applying UC filter for different heights: (a) $15 \mathrm{~km}$, (b) $20 \mathrm{~km}$, (c) $25 \mathrm{~km}$, and (d) $30 \mathrm{~km}$. The range of variable in panel (a) is smaler than others. The coordinates are in kilometers.

(Fig. 6). From the residual maps, shown in Fig. 5, we can identify possible location of oil-gas anomalies. Reservoirs are considered as negative residual anomaly values in the maps. Positive anomalies in 4 corners of the study area (shown in Fig. 6) can be attributed to the rocks of higher density. It is difficult to judge about the geological features of these corners of the study area as there is not enough information about the geology of the area. A re- 
markable zone, identified by a negative anomaly with a north-south extension in the central part of the maps shown in Fig. 6, may be a location for the occurrence of oil-gas reservoirs in the subsurface.

However, a correct interpretation of the maps and accurate determination of existence of oil-gas reservoirs in the subsurface of the area require more information, such as subsurface geological information, well logs, and drilling data from the area. However, this zone, and also other negative anomaly zones, can be considered as the preference zones for the next exploration stages, e.g., seismic surveys or drilling operation. The optimum UC height has been selected as $25 \mathrm{~km}$ based on maximum deflection of the curve presenting correlation between the results of two successive heights of upwarding versus the height (for details see Zeng et al. (2007) and Guo et al. (2013)). The corresponding local or residual anomaly map for the height of $25 \mathrm{~km}$ has been considered for comparison in the next section.

\section{COMPARISON OF THE RESULTS OBTAINED FROM APPLYING FKA AND UC FILTERING METHODS}

For the purpose of comparison of the two filtering methods discussed in this paper, the obtained results of the relevant components from the two methods have been compared together. Therefore, the results of large-scale component of FKA have been compared with the obtained results of applying UC with a large height. Similarly, the results of small-scale component of FKA have been compared with the results of applying UC with a low height. Initially, the regional anomalies obtained from the two methods have been compared together. For the UC method, the regional anomaly component
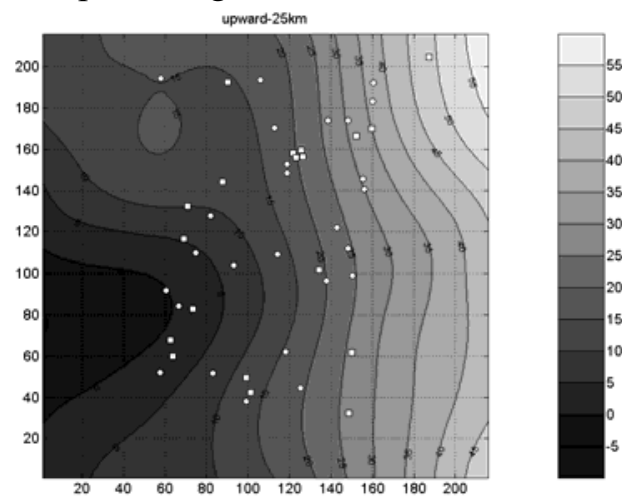
has been extracted from the results of applying optimum UC height of $25 \mathrm{~km}$ to the gravity data (Fig. 7a). In fact, the correct height of upward continuation is qualitatively justified by an expert although dispute on which height 

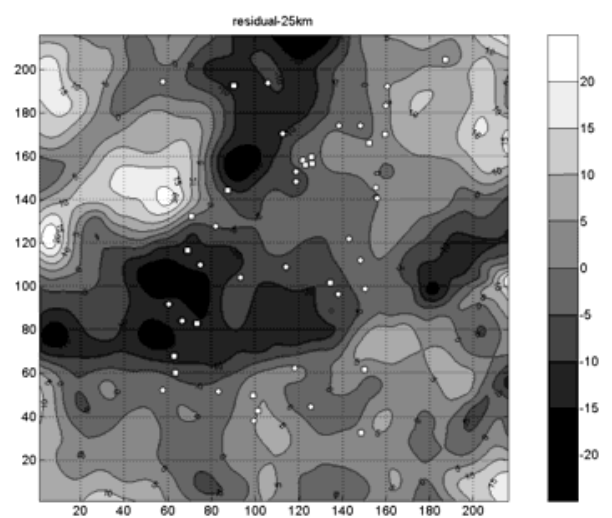

noise + very short-scale componert

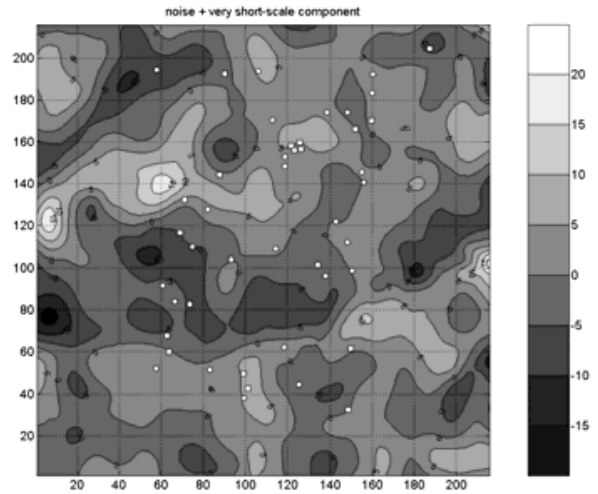

malies obtained from the results of applying the two filtering methods, used in this study, on the gravity data: (a) residual of UC method, (b) short-scale component of FKA method as the first com-ponent of residual, (c) nugget and very short-scale component of FKA method as the second component of residual. The range of value for this variable is different from the local components' range. The coordinates are i

(c)

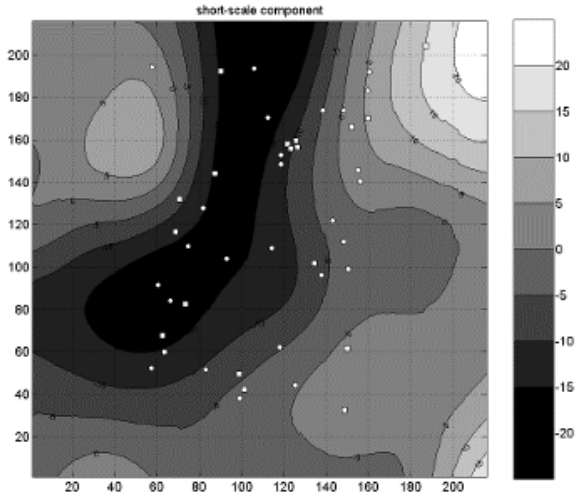

Fig. 7. Comparison of the regional anomalies obtained from the results of applying the two filtering methods, used in this study, on the gravity data: (a) UC method, and (b) FKA method. It should be noted that the range of variable is different in these two panels, but the level of contour is the same for both panels. The coordinates are in kilometers.

(a)

Fig. 8. Comparison of the residual ano-

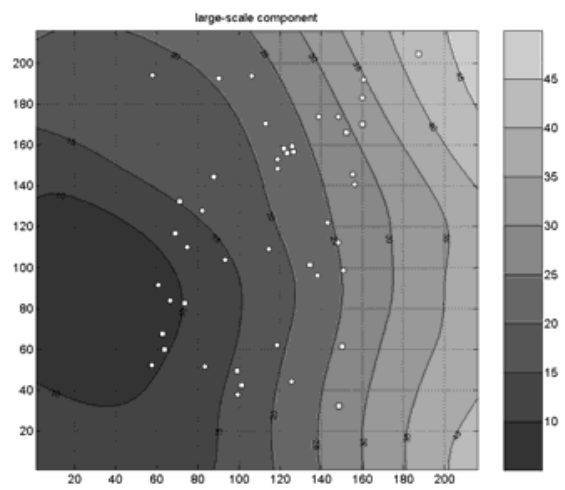

should be the optimum UC height still remains. As can be seen from Fig. 7 , the two maps obtained from the results of FKA and UC methods indicate similar anomalies, trends, and quality, although slight differences between the quantities or values of the two maps are observed. 
The map of short-scale component of FKA and the residual map of UC filter for a height of $25 \mathrm{~km}$ are shown in Fig. 8a. The negative and positive anomalies are situated in almost the same positions in both maps. However, the variable in the FKA map (Fig. 8b) is obviously more continuous than the gravity value of the residual map (Fig. 8a) obtained from the UC method.

The map of the short-scale component of FKA shows a continuous long negative anomaly extended from north to the center and then to the west of the study area. This continuous negative anomaly can be interpreted as a possible long or broad sedimentary zone with a low density compared to the densities of the surrounding rocks. Unlike the FKA map, the residual map shows various negative anomalies or zones in north, center, west, and even east of the study area, implying the existence of various local low density geological features in these parts of the area.

\section{CONCLUSIONS}

Separation of regional and residual anomalies from geophysical data is carried out using various methods. In this paper, the capability of FKA method to separate all the components (not just low-pass and residual as UC) interpretable as regional anomaly (large-scale component) from residual anomaly (short-scale component) in the gravity data from a hydrocarbon field in Vietnam has been demonstrated, and the obtained results have been compared with the results of applying UC filtering method on the gravity data. The UC filtering method is commonly used in gravity and magnetic geophysical methods for separation of regional from local or residual anomalies. We have also shown the superiority of FKA method over UC filter for separation of spatial structures or anomalies. The results of the FKA method depend on the decomposition of the random field model (Bouguer anomaly) into the various structures or factors. The basic difference between the UC and FKA methods is that the FKA method takes into account the spatial structure of data. The geophysical UC filter is often used in trial and error approach on the gravity and magnetic data. In every engineering calculation and modeling, the expert choices will be important and somehow applied in system. FKA is based on spatial structure, and it is possible that two experts choose two different variogram models, but these models could not be much different, as the models are adjusted to experimental variograms calculated directly from data. The FKA method is a multivariate geostatistical method that is applied for the separation of signal from noise based on the recognition of spatial structures with different scales. In addition, the FKA method permits to filter out all of identified structures while this is not applicable in the case of UC method. Furthermore, the sizes and trends of anomalies can be determined from FKA results. Therefore, in addition to general and classic filtering methods, we can also use the FKA method as a strong method in 
filtering spatial structures and anomaly components, and, also, in separation of signal from noise. As the mathematical structure of FKA is similar to ordinary kriging, it can be shown that, in the same way, FKA can handle the problem of clustered data in non-uniform sampled fields, and also is generalized to $n$-dimensional space cases. The results of filtering anomalies in this research work are generally confirmed by geological findings from the study area. However, the accuracy of both FKA and UC methods in filtering anomalies in this case study should be judged when comprehensive or sufficient information becomes available from the subsurface of the area. This information can be obtained from extensive subsurface geological studies, drilling operations, well logging interpretation as well as analyses of cores obtained from numerous wells drilled in the area. Due to the existence of very local changes in the basin characteristics revealed by the closeness of wet and dry wells, a more detailed exploration could be justified in the favorable zones of this basin.

Acknowledgments. We thank Dr. H. Aghajani's Vietnamese teammate, Dr. T.D. Tran, from Vietnamese Academy of Science and Technology, for supplying the gravity data from a hydrocarbon field in southeast Vietnam to us. The authors thank the anonymous reviewers for their constrictive comments.

\section{References}

Aghajani, H. (2009), Investigation of the capabilities of the normalized full gradient method for detecting the hydrocarbon potential areasusing gravity data, Ph.D. Thesis, Shahrood University of Technology, Shahrood, Iran (in Persian).

Aghajani, H., A. Moradzadeh, and H. Zeng (2011), Detection of high-potential oil and gas fields using normalized full gradient of gravity anomalies: A case study in the Tabas basin, eastern Iran, Pure Appl. Geophys. 168, 10, 18511863, DOI: 10.1007/s00024-010-0169-y.

Armstrong, M. (1998), Basic Linear Geostatistics, Springer, Berlin Heidelberg, 155 pp., DOI: 10.1007/978-3-642-58727-6.

Berezkin, W.M. (1973), Application of gravity exploration to reconnaissance of oil and gas reservoir, Neda Publishing House (in Russian).

Chilès, J.-P., and P. Delfiner (1999), Geostatistics: Modeling Spatial Uncertainty, John Wiley \& Sons, New York, 695 pp.

Claerbout, J.F. (1986), Fundamentals of geophysical data processing, Geophys. J. Roy. Astron. Soc. 86, 1 217-219. 
Everett, M.E. (2013), Near-Surface Applied Geophysics, Cambridge University Press, Cambridge.

Galli, A., F. Gerdil-Neuillet, and C. Dadou (1984), Factorial kriging analysis: A substitute to spectral analysis of magnetic data. In: G. Verly, M. David, A.G. Journel, and A. Marechal (eds.), Geostatistics for Natural Resources Characterization. Part 1, D. Reidel Publ. Co., Dordrecht, 543-557, DOI: 10.1007/978-94-009-3699-7_31.

Gibert, D., and A. Galdeano (1985), A computer program to perform transformations of gravimetric and aeromagnetic surveys, Comput. Geosci. 11, 5, 553588, DOI: 10.1016/0098-3004(85)90086-X.

Goovaerts, P. (1997), Geostatistics for Natural Resources Evaluation, Oxford University Press, New York, 483 pp.

Guo, L., X. Meng, Z. Chen, S. Li, and Y. Zheng (2013), Preferential filtering for gravity anomaly separation, Comput. Geosci. 51, 247-254, DOI: 10.1016/ j.cageo.2012.09.012.

Isaaks, E.H., and R.M. Srivastava (1989), An Introduction to Applied Geostatistics, Oxford University Press, New York, 561 pp.

Jacobsen, B.H. (1987), A case for upward continuation as a standard separation filter for potential-field maps, Geophysics 52, 8, 1138-1148, DOI: 10.1190/ 1.1442378 .

Jeannée, N., and J.-L. Mari (2008), Applying geostatistical filtering techniques to near-surface geophysics: two examples for refraction surveying and gravimetry. In: Proc. 70th EAGE Conference \& Exhibition, 9-12 June 2008, Rome, Italy, $5 \mathrm{pp}$.

Magneron, C., M. Bourges, and N. Jeannée (2009), M-Factorial Kriging for seismic data noise attenuation. In: 11th Int. Congr. Brazilian Geophysical Society and EXPOGEF 2009, 24-28 August 2009, Salvador, Brazil, 1651-1654.

Matheron, G. (1982), Pour une analyse krigeante des données régionalisées, Report N-732, Centre de Géostatistique, Fontainebleau, France (in French).

McCulloh, T.H. (1980), Mass properties of sedimentary rocks and gravimetric effects of petroleum and natural-gas reservoirs. In: D. Guion and C. Prieto (eds.), Gravity Magnetic Exploration Applications, Society of Exploration Geophysicists.

Nettleton, L.L. (1954), Regional, residuals, and structures, Geophysics 19, 1, 1-22, DOI: $10.1190 / 1.1437966$.

Que, B.C., and T.D. Tran (1996), Geodynamics and mineral potential of the offshore area of Vietnam, Final report KT-03-02, Marine Research Program, Hanoi, Vietnam.

Reynolds, J.M. (1997), An Introduction to Applied and Environmental Geophysics, John Wiley \& Sons, 806 pp.

Sandjivy, L. (1984), The factorial kriging analysis of regionalized data: Its application to geochemical prospecting. In: G. Verly, M. David, A.G. Journel, and 
A. Marechal (eds.), Geostatistics for Natural Resources Characterization. Part 1, D. Reidel Publ. Co., Dordrecht, 559-571, DOI: 10.1007/978-94009-3699-7 32.

Sandjivy, L., and A. Galli (1984), Kriging analysis versus spectral analysis, Inform. Geol. 21, 115-124.

Séguret, S.A. (1993). Analyse krigeante spatio-temporelle appliquée à des données aéromagnétiques, Cah. Géostat. 3, 115-138 (in French).

Tan, T.K., and N.Q. Bo (2002), The main structural elements in Cenozoic of the continental shelf of Vietnam. In: Proc. Scientific Conference "Bien Dong 2002", 16-19 September 2002, Nha Trang, Vietnam.

Telford, W.M., L.P. Geldart, and R.E. Sheriff (1990), Applied Geophysics, 2nd ed., Cambridge University Press, New York.

Tran, D.T. (2004), Two and three dimensional normalized total gradient of gravity anomalies and its application for detecting the oil-gas potential areas in the southeast basins of the East Vietnam Sea. In: 7th SEGJ Int. Symp. "Imaging Technology", 24-26 November 2004, Sendai, Japan, 1-6.

Vu, N.G. (2003), Structural evolution of the block 102 and 106 Red river basinimplication for hydrocarbon potential. In: Proc. Conf. Vietnam Petroleum Institute: 25 Years of Development and Achievement, 283-309.

Wackernagel, H. (1988), Geostatistical techniques for interpreting multivariate spatial information. In: C.F. Chung, A.G. Fabbri, and R. Sinding-Larsen (eds.), Quantitative Analysis of Mineral and Energy Resources, NATO ASI Series, Vol. 223, D. Reidel Publishing Co., 393-409, DOI: 10.1007/978-94009-4029-1_24.

Wackernagel, H. (1995), Multivariable Geostatistics: An Introduction with Applications, Springer, Berlin Heidelberg, 387 pp.

Yao, T. (2004), Reproduction of the mean, variance, and variogram model in spectral simulation, Math. Geol. 36, 4, 487-506, DOI: 10.1023/B:MATG. 0000029301.22150 .28 .

Zeng, H., X. Meng, C. Yao, X. Li, H. Lou, Z. Guang, and Z. Li (2002), Detection of reservoirs from normalized full gradient of gravity anomalies and its application to Shengli oil field, east China, Geophysics 67, 4, 1138-1147, DOI: 10.1190/1.1500375.

Zeng, H., D. Xu, and H. Tan (2007), A model study for estimating optimum upwardcontinuation height for gravity separation with application to a Bouguer gravity anomaly over a mineral deposit, Jilin province, northeast China, Geophysics 72, 4, I45-I50, DOI: 10.1190/1.2719497.

Received 12 February 2014

Received in revised form 22 December 2014

Accepted 5 January 2015 